K. Jung mentioned, we did not evaluate the influence of blood sampling and separation of serum from blood cells. We agree with $\mathrm{M}$. John and $\mathrm{K}$. Jung that we must be aware of pre-analytical problems when analysing the circulating matrix metalloproteinase (MMP)-9 and tissue inhibitor of metalloproteinase (TIMP)-1 levels. However, we used only serum samples in our study because we usually use serum samples for clinical examinations in our hospital. Therefore, we think that the comparison of the serum TIMP-1 and MMP-9 concentrations in each patient group is valid. Our results are consistent with serum TIMP-1 and MMP-9 concentrations reported elsewhere $[2,3]$.

We also agree with $\mathrm{M}$. John and K. Jung that the changes of white blood cell count during chronic obstructive pulmonary disease (COPD) exacerbations could have influenced TIMP-1 concentrations, because the white blood cell count increased during most exacerbations. We are now planning to identify specific cell types that are involved in the production and secretion of circulating TIMP-1 and MMP-9 in COPD patients.

We thank M. John and K. Jung for their suggestions for our future studies.

\section{Y. Higashimoto}

Dept of Internal Medicine, Wakayama Medical University Kihoku Hospital, Ito-gun, Japan.

\section{REFERENCES}

1 Higashimoto $\mathrm{Y}$, Yamagata $\mathrm{Y}$, Iwata $\mathrm{T}$, et al. Increased serum concentrations of tissue inhibitor of metalloproteinase-1 in COPD patients. Eur Respir J 2005; 25: 885-890.

2 Bosse M, Chakir J, Rouabhia M, Boulet LP, Audette M, Laviolette M. Serum matrix metalloproteinase-9: tissue inhibitor of metalloproteinase-1 ratio correlates with steroid responsiveness in moderate to severe asthma. Am J Respir Crit Care Med 1999; 159: 596-602.

3 Oshita Y, Koga T, Kamimura T, Matsuo K, Rikimaru T, Aizawa H. Increased circulating $92 \mathrm{kDa}$ matrix metalloproteinase (MMP-9) activity in exacerbations of asthma. Thorax 2003; 58: 757-760.

\title{
ECG for risk stratification in patients with pulmonary embolism
}

\section{To the Editors:}

We read with interest the paper by GEIBEL et al. [1] on the prognostic value of the ECG in patients with acute major pulmonary embolism (PE). In the last few years a number of studies have been published for risk stratification of patients with PE, with the suspicion that there could be a subgroup of haemodynamically stable patients (submassive PE), in whom thrombolysis could be beneficial [2-4].

The hypothesis of the study [1] was that ECG could be a simple baseline test (as compared with echocardiography) to identify risk of death in patients with PE. However, the population of the study included either patients with haemodynamically unstable PE, in whom thrombolysis is usually indicated, or patients with submassive PE, who are identified by echocardiographic findings. Therefore, the results may be of limited value in clinical practice.

We studied 302 consecutive normotensive patients with a diagnosis of PE in a 2-yr period. The mean age was $68 \mathrm{yrs}(95 \%$ confidence interval (CI): 66-70) of whom $55 \%$ were female. We analysed the prognostic relevance of ECG with respect to early mortality (defined as those presented in the first 30 days). ECG was available in 278 patients, of which 116 (42\%) were normal. ECG abnormalities were: 1) sinus tachycardia in 93 patients; 2) ST-T abnormalities in 29 patients; 3) complete right bundle branch block in 42 patients; 4) S1Q3T3 pattern in 32 patients; 5) atrial arrythmia in 22 patients; and 6) right axis pattern in two patients. Early death occurred in 16 patients $(6 \%)$. The 12-lead ECG did not show differences between survivors and nonsurvivors during the first 30 days after admission. Univariate analysis revealed that ECG abnormalities were not significant independent predictors of outcome (odds ratio: 0.7; 95\% CI: 0.2-2.5). Our results do not support the usefulness of ECG for risk stratification in haemodynamically stable patients with pulmonary embolism.

\section{Jimenez \\ Ramon y Cajal Hospital, Madrid, Spain.}

\section{REFERENCES}

1 Geibel A, Zehender M, Kasper W, Olschewski M, Klima C, Konstantinides SV. Prognostic value of the ECG on admission in patients with acute major pulmonary embolism. Eur Respir J 2005; 25: 843-848.

2 Konstantinides S, Geibel A, Heusel G, et al. Heparin plus alteplase compared with heparin alone in patients with submassive pulmonary embolism. N Engl J Med 2002; 347: 1143-1150.

3 Giannitsis E, Muller-Bardorff M, Kurowski V, et al. Independent prognostic value of cardiac troponin $\mathrm{T}$ in 
patients with confirmed pulmonary embolism. Circulation 2000; 102: 211-217.

4 Pruszczyk P, Bochowicz A, Torbicki A, et al. Cardiac troponin $\mathrm{T}$ monitoring identifies high-risk group of normotensive patients with acute pulmonary embolism. Chest 2003; 123: 1947-1952.

DOI: $10.1183 / 09031936.05 .00054805$

\section{From the authors:}

As D. Jimenez correctly pointed out, our study [1] was based on a large registry, which focused on patients with major, i.e. massive or submassive, pulmonary embolism (PE). This is the critical patient population in which not only confirmation of PE itself, but also, particularly a high level of suspicion and the prompt diagnosis (or exclusion) of right ventricular dysfunction may have enormous prognostic and therapeutic implications. We could show that the presence of at least one of pre-specified ECG abnormalities on admission was, apart from the clinical findings at presentation, a predictor of outcome in the acute phase. The message of our study is, therefore, that the
ECG may be useful as a simple, noncostly initial (baseline) tool for assessing the patients' prognosis and for guiding further diagnostic work-up. We did not claim that the ECG should, or could, be used as an alternative to imaging studies, such as echocardiography or helical computed tomography, to diagnose RV dysfunction, or that ECG changes, such as the ones we examined could, by themselves, suffice to set the indication for thrombolytic treatment in PE.

Rather, the value of a pathological ECG lies in the fact that it may help a large number of clinicians, regardless of their specialty, suspect and seek to confirm right ventricular dysfunction more frequently than is presently the case.

\section{S. Konstantinides}

University of Goettingen, Goettingen, Germany.

\section{REFERENCES}

1 Geibel A, Zehender M, Kasper W, Olschewski M, Klima C, Konstantinides SV. Prognostic value of the ECG on admission in patients with acute major pulmonary embolism. Eur Respir J 2005; 25: 843-848.

DOI: $10.1183 / 09031936.05 .00060505$ 